\title{
PARP Inhibition Prevents Ethanol-Induced Neuroinflammatory Signaling and Neurodegeneration in Rat Adult-Age Brain Slice Cultures
}

\author{
Nuzhath Tajuddin, Hee-Yong Kim, and Michael A. Collins
}

Department of Molecular Pharmacology and Therapeutics, Stritch School of Medicine, Loyola University Chicago, Maywood, Illinois (N.T.; M.A.C.) and Laboratory of Molecular Signaling, National Institute of Alcoholism and Alcohol Abuse, National Institutes of Health, Bethesda, Maryland (H.-Y.K.)

Received September 22, 2017; accepted December 13, 2017

\begin{abstract}
Using rat adult-age hippocampal-entorhinal cortical (HEC) slice cultures, we examined the role of poly [ADP-ribose] polymerase (PARP) in binge ethanol's brain inflammatory and neurodegenerative mechanisms. Activated by DNA strand breaks, PARP (principally PARP1 in the brain) promotes DNA repair via poly [ADP-ribose] (PAR) products, but PARP overactivation triggers regulated neuronal necrosis (e.g., parthanatos). Previously, we found that brain PARP1 levels were upregulated by neurotoxic ethanol binges in adult rats and HEC slices, and PARP inhibitor PJ34 abrogated slice neurodegeneration. Binged HEC slices also exhibited increased $\mathrm{Ca}^{+2}$-dependent phospholipase $\mathrm{A} 2$ (PLA2) isoenzymes (cPLA2 IVA and SPLA2 IIA) that mobilize proinflammatory $\omega 6$ arachidonic acid (ARA). We now find in 4-day-binged HEC slice cultures (100 mM ethanol) that PARP1 elevations after two overnight binges precede PAR, CPLA2, and sPLA2 enhancements by 1 day and high-mobility group box-1
\end{abstract}

(HMGB1), an ethanol-responsive alarmin that augments proinflammatory cytokines via toll-like receptor-4 (TLR4), by 2 days. After verifying that PJ34 effectively blocks PARP activity $(\uparrow P A R)$, we demonstrated that, like PJ34, three other PARP inhibitors-olaparib, veliparib, and 4-aminobenzamide-provided neuroprotection from ethanol. Importantly, PJ34 and olaparib also prevented ethanol's amplification of the PLA2 isoenzymes, and two PLA2 inhibitors were neuroprotectivethus coupling PARP to PLA2, with PLA2 activity promoting neurodegeneration. Also, PJ34 and olaparib blocked ethanolinduced HMGB1 elevations, linking brain PARP induction to TLR4 activation. The results provide evidence in adult brains that induction of PARP1 may mediate dual neuroinflammatory pathways (PLA2 $\rightarrow$ phospholipid $\rightarrow$ ARA and HMGB1 $\rightarrow$ TLR $4 \rightarrow$ proinflammatory cytokines) that are complicit in binge ethanol-induced neurodegeneration.

\section{Introduction}

Severe alcohol (ethanol) abuse and chronic binge alcoholism impair brain neuronal and glial pathways associated with memory and cognition. Consequently, ethanol abuse/ addiction is a relatively widespread-yet surprisingly underacknowledged - reason for cognitive impairment or decline and dementia worldwide (Ilomaki et al., 2015; Sachdeva et al., 2016). We and others have linked neuroinflammatory mechanisms to brain damage in ethanol-binged adult rodent models. Our laboratory has focused on phospholipid-based pathways (Brown et al., 2009; Collins and Neafsey, 2012), specifically phospholipase A2 (PLA2) enzymes that govern the mobilization and availability of $\omega 6$ arachidonic acid (ARA), an often proinflammatory precursor molecule and of antiinflammatory $\omega 3$ docosahexaenoic acid (DHA), both of which

The research is supported by National Institutes of Health [Grant U01 AA018279] (M.A.C.) and Loyola University Chicago Research Committee.

https://doi.org/10.1124/jpet.117.245290. are present in the mammalian brain in approximately equivalent amounts. Also of interest have been the possible role and association of aquaporin-4 (AQP4) water channels that stimulate brain edema (which also develops in ethanolbinged rats), increase PLA2 activities, and augment cytokines such as tumor necrosis factor when enhanced by ischemia or trauma. With respect to this latter proinflammatory cytokine and related "kines," other laboratories have coupled ethanol's neuroinflammatory effects to increased cytokines, resulting via neuroimmune/inflammasome pathways involving toll-like receptors (especially TLR4) and brain danger signals or alarmins-notably, high mobility group box-1 (HMGB1).

We initially observed that comparatively moderate binge ethanol treatment ( $\sim 3 \mathrm{~g} / \mathrm{kg}$ per day) of male rats as juveniles and young adults promoted elevations of an important DNAinteracting enzyme, poly [ADP-ribose] polymerase-1 (PARP1), in brain regions particularly susceptible to ethanol-induced neurodegeneration (Tajuddin et al., 2013). Associated with the

ABBREVIATIONS: AQP4, aquaporin-4; ARA, arachidonic acid; ATA, aristolochic acid; ATK, arachidonoyl trifluoromethyl ketone; cPLA2, Ca ${ }^{+2}{ }_{-}$ dependent cytosolic phospholipase A2; DHA, docosahexaenoic acid; HEC, hippocampal-entorhinal cortical complex; HMGB1, high-mobility group box 1; HS, horse serum; MEM, minimum Eagle's medium; OCM, one-carbon metabolism; PARP, poly [ADP-ribose] polymerase; PI, propidium iodide; RAGE, receptor for advanced glycation end products; ROS, reactive oxygen species; SPLA2, Ca ${ }^{+2}$-dependent secreted PLA2; TLR4, tolllike receptor-4. 
PARP1 elevations were regionally selective increases of two $\mathrm{Ca}^{+2}$-dependent PLA2 enzymes responsible for ARA liberation from membrane phospholipids-cytosolic PLA2 (cPLA2 IVA; also termed cPLA2 $\alpha$ ), phosphorylated (activated) cPLA2 IVA, and secreted PLA2 (sPLA2 IIA). Reductions in $\mathrm{Ca}^{+2}$. independent PLA2 (iPLA2 VIA), a regulator of mitochondrial function (Kinghorn et al., 2015) and brain $\omega 3$ docosahexaenoic acid dynamics (Cheon et al., 2012), also occurred, along with regional upregulation of $\mathrm{AQP} 4$ water channels. More recently, similar alterations of PARP1, the preceding PLA2 isozymes, and AQP4 selectively occurred in the hippocampus and entorhinal cortex of severely binged adult male rats (Tajuddin et al., 2014) — that is, the Majchrowicz binge model, which undergoes neurodegeneration chiefly in these two regions (Collins et al., 1996) and displays cognitive impairments (Obernier et al., 2002b). In parallel with the preceding in vivo experiments, repeated ethanol treatment of adult rat organotypic hippocampal-entorhinal cortical (HEC) slice cultures produced corresponding PARP1, PLA2, and AQP4 changes.

This article reports pharmacologic studies using adult HEC slice cultures to examine the possibility that excessive PARP1 activation engendered by binge ethanol exposures mediates dual neuroinflammatory phospholipid-ARA and neuroimmune HMGB1-cytokine pathways leading to neuronal damage - fundamentally, a pleiotropic master inflammatory regulator in brain. A growing literature confirms that, upon hyperinduction from DNA strand breaks arising from pro-oxidative brain insults (i.e., stroke or trauma), PARP1 and its PAR products elicit neuroinflammation and neurodegeneration (Narne et al., 2017). Mechanisms proposed for PARP1-triggered neuronal impairments include neuronal energy/ATP deficits from PARP-dependent depletion of $\mathrm{NAD}+$ and/or PAR inhibition of hexokinase (glycolysis) and PAR-initiated mitochondrial release of apoptosis-inducing factor-which, diverging from its name, promotes nuclear chromatin damage and a regulated necrosis termed parthanatos (David et al., 2009).

Current experimental studies of ethanol and brain PARP have used primarily fetal and developmental rat models (Cherian et al., 2008; Gavin et al., 2016). With respect to neuroinflammation, brain PARP has been reported to stimulate cyclooxygenase-2 (Koh et al., 2004), an important enzyme in ARA metabolism that is responsive to ethanol (Knapp and Crews, 1999; Valles et al., 2004), but to our knowledge no connection has been reported with neurodegeneration models between PARP and key isoforms of PLA2, the rate-limiting process liberating ARA for cyclooxygenases. Thus, in addition to results verifying that PARP (and, most likely, the predominant brain form, PARP1) is important in binge ethanolrelated neurotoxicity, we provide evidence that PARP amplifies PLA2 isozymes that mobilize ARA, which would generate a plethora of neuroinflammatory lipid mediators and reactive oxygen species (ROS) that can cause neurodamage, and our results with two PLA2 inhibitors support this possibility. Furthermore, consistent with evidence using non-neuronal cells that couples PARP1 activation to the endogenous TLR4 agonist, HMGB1 (Ditsworth et al., 2007; Yang et al., 2014), our results suggest that the overall levels of this ethanol-responsive danger signal for proinflammatory cytokine stimulation (Crews et al., 2017) are regulated by PARP in the adult brain.

\section{Materials and Methods}

Inhibitors and Antibodies. Olaparib and veliparib were obtained from Selleckchem (Houston, TX), and PJ34 was from Enzo Life Sciences (Ann Arbor, MI). Arachidonoyl trifluoromethyl ketone (ATK) and aristolochic acid (ATA) were from Sigma-Aldrich (St. Louis, MO). Santa Cruz Biotechnology (Santa Cruz, CA) provided antibodies for cPLA2 IVA (1:1000, sc-454), AQP4 (1:1000, sc-20812), and GAPDH (1:2000, sc-34391); Millipore (Temecula, CA) was the source of iPLA2 VIA antibody (1:1000, 07-169-1). Antibodies for sPLA2 IIA (1:500, 1811055100) was obtained from BioVendor (Candler, NC), and PAR antibody (1:500, BML-SA216.0100) was from Enzo Life Sciences; all were rabbit except PAR (mouse) and cPLA2 (mouse). Secondary antibodies (1:10,000) were from Jackson ImmunoResearch (West Grove, PA), and luminol reagent for immunoblot detection was from Pierce Chemicals (Rockford, IL). Other chemicals and reagents were purchased from Sigma Chemical Co. (St. Louis, MO).

Preparation of Rat Adult-Age HEC Slice Cultures. Based on a Loyola University Medical Center Institutional Animal Care and Use Committee -approved protocol, 40- to 42-day old male SpragueDawley rats were anesthetized with isoflurane and thoroughly perfused via an intracardiac catheter with $150 \mathrm{ml}$ ice-cold lactateRinger's solution. Translucent brains were removed and placed in icecold Gey's buffer solution. The HEC was isolated and 250- to $300-\mu \mathrm{m}$ slices were prepared with a McIlwain tissue chopper. Slices were transferred carefully to sterile Millicell-CM membrane inserts $(0.4-\mu$ pore size, 30-mm diameter, $\sim 3$ slices/insert; Millipore Corp) in six-well plastic plates. Each well contained $1.2 \mathrm{ml}$ of minimum Eagle's medium (MEM)/25\% heat-inactivated horse serum (HS) containing $6.5 \mathrm{mg} / \mathrm{ml}$ glucose, a volume that saturates insert membranes but does not cover slices. Plates with inserts + slices were then incubated in $5 \% \mathrm{CO}_{2}$ at $32^{\circ} \mathrm{C}$; after 48 hours, plates were transferred to a $37^{\circ} \mathrm{C}$ incubator at $5 \%$ $\mathrm{CO}_{2}$, with a complete media change (but with $20 \% \mathrm{HS}$ ), and then cultured as such for 16-18 days with media changes every 3 days. Slices were visually monitored throughout, and those appearing abnormally dark or with darkened regions were discarded.

Ethanol Treatments and Neurodegeneration and Immunoblot Assays. At brain ages of $62 \pm 2$ days (18-20 days in culture), slices in experimental groups were treated over four successive overnight intervals ( 16 hours) with $100 \mathrm{mM}$ ethanol in MEM/20\% $\mathrm{HS}$ and to the complete media without ethanol during subsequent daylight hours ( $~ 8$ hours) for 3 of the 4 days. The binge exposure, designed to mirror repetitive binge intoxication in severe, chronic alcoholism, has been shown to promote HEC neuroinflammation and neuronal damage in this in vitro model (Collins et al., 2013; Tajuddin et al., 2014). Control slices were treated similarly throughout to changes of ethanol-free complete media. At concentrations stated, PARP inhibitors or PLA2 inhibitors were added 2 hours before ethanol + media or media only and were present throughout the 4 nights and interior 3 days. The morning of day 4, all slices were allowed $\sim 4$ hours in freshly added media, less inhibitors and ethanol, before propidium iodide (PI) analysis or preparation for immunoblot assays. In the time-course studies (Fig. 1) that did not include inhibitors, respective immunoblot analyses were done after $\sim 4$ hours on the specific day indicated.

For PI staining, media were discarded, and slices were incubated with $1.2 \mathrm{ml}$ of fresh serum-free media containing $10 \mu \mathrm{M}$ PI, a widely used fluorescent stain used with neurotoxin-treated brain slice cultures to quantify dead or dying neurons (Collins et al., 2013). After 25-30 minutes, media were removed, and slices were rinsed twice with fresh serum-free media, followed by the addition of MEM/20\% HS for 1 hour before observation of slice fluorescence results of PI-stained cells. Fluorescence was quantitated with 1.99 National Institutes of Health Image J software using a Nikon DS-5 camera + Nikon TS100 inverted microscope. The percentage of slice area with PI fluorescence above background was calculated in relation to the total area for each slice; the same threshold level was used for all slices in an experiment. 


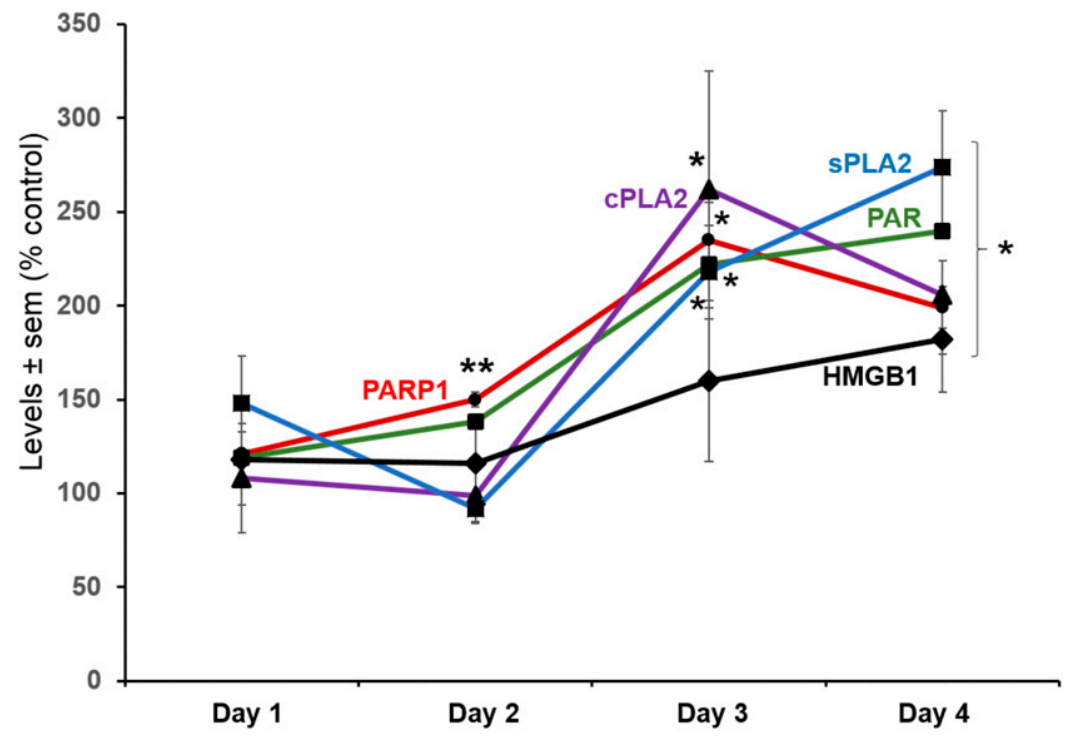

Fig. 1. Time course for PARP1, PAR, cPLA2, sPLA2, and HMGB1 levels in adult rat organotypic HEC slice cultures throughout 4 days of binge ethanol (E) exposure reveal temporal priority of PARP1. Slice levels of PARP1 were increased significantly above control levels beginning after the second overnight $\mathrm{E}$ exposure. Slice levels of PAR, cPLA2 IVA, and sPLA2 IIA were augmented significantly above respective control levels beginning after the third overnight E exposure. Slice levels of HMGB1 were increased significantly above control levels beginning after the fourth overnight $\mathrm{E}$ exposure $(* * P \leq 0.001 ; * P \leq 0.05 ; n=3-5)$.

For immunoblot analyses, slices were rinsed twice with $1 \times$ phosphate-buffered saline, pooled (six to eight slices) in lysis buffer ( $0.1 \%$ SDS, $1 \%$ sodium doxycholate, $1 \%$ Triton X-100, $150 \mathrm{mM} \mathrm{NaCl}$, and $25 \mathrm{mM}$ Tris $\mathrm{HCl}$ ) containing protease and phosphatase inhibitors, sonicated for 15 seconds, and centrifuged 10 minutes at 13,000 rpm. Supernatants were removed, and aliquots were taken for protein assays (BCA method; Pierce Biotech., Rockford, IL). Additional aliquots (30-40 $\mu \mathrm{g}$ protein/aliquot) were then separated by $4 \%-20 \%$ SDS-PAGE, transferred to nitrocellulose membranes, and treated with respective protein or PAR antibodies. Intensities of immunoblots were normalized to GAPDH, obtained on the same gels after stripping and reprobing, and images were scanned with LABwork 4.5 image acquisition and analysis software (UltraViolet Products, Upland, CA). Immunoblot levels were determined relative to levels of GAPDH as housekeeping protein and expressed as percent of control levels (mean \pm S.E.M.)

Statistical Analyses. For immunoblots, one $n$ contains 8-10 pooled slices (two wells). For PI analyses, the number of slices is stated. Results were analyzed for statistically significant differences $(P<0.01$ or $P<0.05)$ by Tukey's test and one-way analysis of variance with completely randomized design.

\section{Results}

Ethanol Significantly Increases PARP1 before Causing Elevations of Neuroinflammation-Associated Proteins cPLA2, SPLA2, and HMGB1. In our previous binge ethanol experiments with adult HEC slice cultures, PARP1 was significantly increased after four sequential overnight binge exposures, in concert with PAR, cPLA2, and sPLA2. To obtain insight into the possible temporal signaling involving these species as well as the alarmin protein, HMGB1, we ascertained with immunoblots the daily time courses of significant changes in HEC slice levels resulting from ethanol binges. As shown in Fig. 1, PARP1 preceded the other species in being significantly elevated after the second overnight ethanol exposure (day $2 ; F(1: 5)=64.1, P=0.001$ ), and it continued to escalate on days $3(P=0.05)$ and $4(P=0.05)$. Levels of its PAR product, although apparently higher than control on day 2 , were slightly outside statistical significance $(P=\sim 0.06)$ at this juncture; however, after the third and fourth ethanol exposures, PAR levels were significantly elevated (day $3: F(1: 5)=10.97, P=0.03$; day
4: $F(1: 5)=9.58, P=0.036)$. Also significantly above their respective control levels by days 3 and 4 were both cPLA2 IVA (day 3: $F(1: 7)=5.985, P=0.05$; day $4: F(1: 7)=15.45$, $P=0.008$ ) and sPLA2 IIA (day $3: F(1: 9)=10.794, P=0.011$; day $4: F(1: 9)=6.63, P=0.033)$. Not until the 4th day of ethanol exposure, however, two binge exposure periods after PARP1 elevations and one ethanol exposure after increases of PAR and the $\mathrm{Ca}^{+2}$-dependent PLA2 enzymes, were HMGB1 levels significantly elevated above control values $(F(1: 7)=$ $6.53, P=0.043$ ).

Neuroprotective PJ34 Dose Inhibits EthanolDependent PARP Activity (PAR Levels) and Three Other PARP Inhibitors Neuroprotect from EthanolLike PJ34. To verify that by increasing PARP1 ethanol does enhance PARP activity and that the neuroprotective effectiveness of PJ34 involves curbing elevated activity, we assayed slice PAR levels by immunoblot after 4 days of binge ethanol. For immunoblot quantitation, integrated densities of the entire lanes, which encompass 50 - and $125-\mathrm{kDa}$ parylated species and other faint PAR-associated macromolecular species as expected, were taken. Figure 2 shows that ethanol augmented the PAR species $(F(3: 15)=7.785, P=0.004)$ and PJ34 effectively blocked the ethanol-induced elevations of $\operatorname{PAR}(F(3: 15)=7.785, P=0.001)$. To establish definitively the connection between PARP and ethanol neurotoxicity in the HEC slice cultures, we examined the effects on neurodegeneration from 4 days of ethanol binges of three other PARP inhibitors, as evidenced by PI staining. In Fig. 3A, ethanol induced significant neurodegeneration $(F(5: 119)=3.79$, $P=0.003$ ) and olaparib, a selective PARP inhibitor approved by the Food and Drug Administration for cancer cotherapy treatment, significantly counteracted the ethanol-elicited neurodegeneration at both $0.1(P=0.004)$ and $1 \mu \mathrm{M}$ concentrations $(P=0.008)$. Similarly, as shown in Fig. 3B, the neurodegeneration caused by 4 days of ethanol $(F(5: 100)=$ $9.85, P=0.01$ ) was completely prevented by veliparib, a another selective chemotherapy-used PARP inhibitor, at 0.1 $(P=0.05)$ and $1 \mu \mathrm{M}(P=0.001)$. Furthermore, Fig. 3C shows that the ethanol-induced neurodegeneration $[F(3: 29)=7.76$, $P=0.01]$ was reduced by $\sim 60 \%$ by an older and less specific PARP inhibitor, 4-aminobenzamide $(4-\mathrm{AB})(P=0.05)$, at 


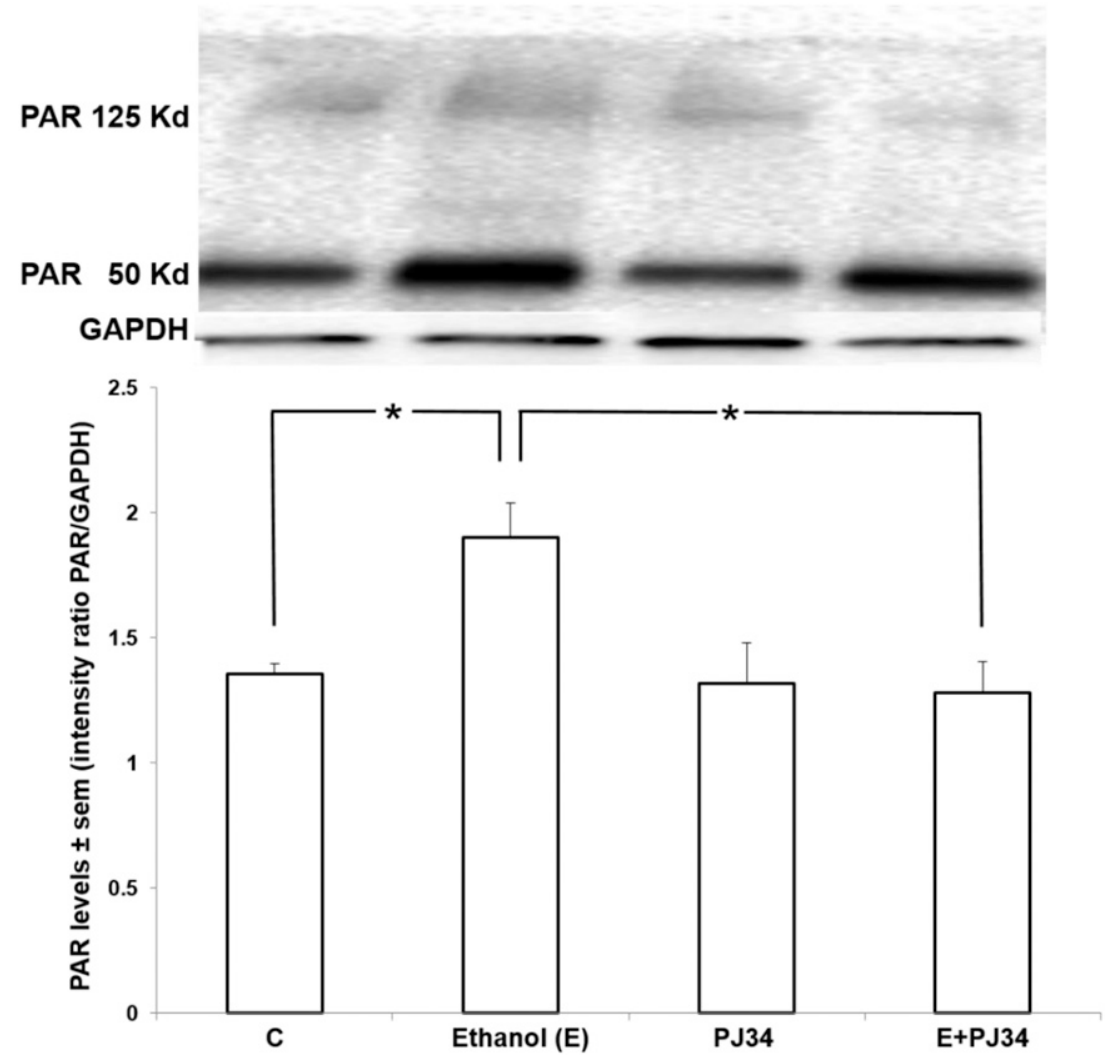

Fig. 2. PARP inhibition by PJ34 suppresses binge ethanol (E)-induced PAR elevations in adult rat HEC slice cultures. Overnight exposure of HEC slice cultures to $\mathrm{E}(100 \mathrm{mM})$ for 4 days significantly increased slice levels of PAR products, and cotreatment throughout with PJ34 (10 $\mu \mathrm{M})$ abolished the increased PAR content in ethanol-binged HEC slices. ${ }^{*} P \leq 0.01$, E vs. control (C) or E vs. $\mathrm{E}+\mathrm{PJ} 34(n=4)$
$500 \mu \mathrm{M}$. In Fig. 3D, representative images of PI-stained slices display the fluorescent density in a binge ethanol-exposed slice, and diminished densities in images for $\mathrm{E}$ cotreated with the above three PARP inhibitors-olaparib (1 $\mu \mathrm{M})$, veliparib $(1 \mu \mathrm{M})$, and 4-AB $(500 \mu \mathrm{M})$. Not shown for convenience are slices containing only the PARP inhibitors, which show negligible PI staining that is indistinguishable from control slices.

PARP Inhibitors Counteract Ethanol-Induced Elevations of cPLA2 and sPLA2 but Not Reductions of iPLA2. In previous immunoblot assays of extracts of adult HEC slice cultures undergoing neurotoxic ethanol binges, we found significantly elevated cPLA2 IVA and sPLA2 IIA, two $\mathrm{Ca}^{+2}$-dependent enzymes that have vital roles in mobilization of ARA, which is often neuroinflammatory and pro-oxidative, and significant reductions in a major brain $\mathrm{Ca}^{+2}$-independent PLA2 (iPLA2 VIA). As connections between brain PARP and PLA2 proteins have not been reported, we explored whether PARP inhibition interfered with binge ethanol's effects on these key isoenzymes. The results, shown in Fig. 4A, confirmed that ethanol promoted significant cPLA2 IVA elevations $(F(3: 53)=4.7, P=0.05)$ and show for the first time that PJ34 cotreatment ( $10 \mu \mathrm{M})$ effectively prevented the increases $(P=0.04)$. Furthermore, Fig. $4 \mathrm{~B}$ shows that augmentation of ethanol-induced cPLA2 IVA $(F(3: 24)=2.97, P=0.04)$ was also blocked by the more selective olaparib at $1 \mu \mathrm{M}(P=$ 0.043). (Although olaparib at the 10 -fold lower, neuroprotective $0.1-\mu \mathrm{M}$ dose reduced cPLA2 IVA to about control values, statistical significance versus ethanol-induced levels was not obtained $(P=0.08)$ ). With sPLA2 IIA, Fig. $4 \mathrm{C}$ shows that, as with cPLA2, ethanol-induced increases in this sPLA2 isoform $(F(3: 31)=11.91, P=0.001)$ that we reported earlier were completely prevented by $10 \mu \mathrm{M}$ PJ34 $(P=0.004)$. Similarly, in Fig. 4D, ethanol-induced enhancement of sPLA2 levels over control $(F(3: 15)=4.1, P=0.032)$ were blocked by $1 \mu \mathrm{M}$ olaparib $(P=0.044)$. In contrast to the counteracting effect of PARP inhibition on cPLA2 and sPLA2 elevations, the reduction in levels of iPLA2 VIA caused by 4 days of ethanol binges that we have observed in vivo and shown in Fig. 4E $(F(3: 11)=5.9, P=0.05)$ was not significantly reversed by $10 \mu \mathrm{M}$ PJ34 $(P=0.72)$. Likewise, Fig. $4 \mathrm{~F}$ shows that the significantly reduced brain slice iPLA2 VIA owing to binge ethanol $(F(1,12)=6.35, P=0.028)$ was not prevented by $1 \mu \mathrm{M}$ olaparib $(P=0.152)$.

Inhibitors of cPLA2 and sPLA2 Are Highly Neuroprotective against Binge Ethanol. Pharmacologic examination of the importance of PARP-upregulated cPLA2 and sPLA2 in binge ethanol-induced neurodegeneration was done with adult HEC slice cultures using the relatively specific inhibitors of these two phospholipase classes, ATK and ATA, respectively. In Fig. 5A, ethanol's promotion of neurodegeneration $(F(5: 129)=16.77, P=0.001)$ was significantly reduced $\sim 45 \%$ by coexposure with $5 \mu \mathrm{M}$ of cPLA2 inhibitor, ATK $(P=$ 03 ), whereas at $25 \mu \mathrm{M}, \mathrm{ATK}$ provided essentially complete neuroprotection $(P=0.001)$. In Fig. 5B, the significant increase in ethanol-elicited neurodegeneration $(F(5: 139)=$ $33.4, P=0.001$ ) was completely blocked by cotreatment with the sPLA2 inhibitor ATA at both $50(P=0.001)$ and $500 \mu \mathrm{M}$ $(P=0.001)$.

PARP Inhibition Does Not Obstruct Ethanol-Induced Upregulation of AQP4. In our previously reported HEC slice and in vivo experiments, ethanol binges over 4 or more days promoted significant enhancements of AQP4 levels, concomitant with brain edema and neurodegeneration 
A

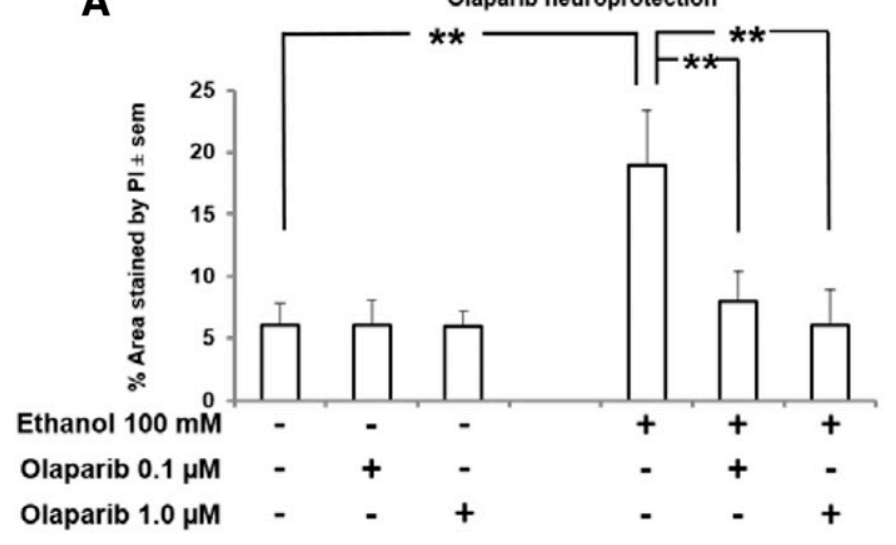

B

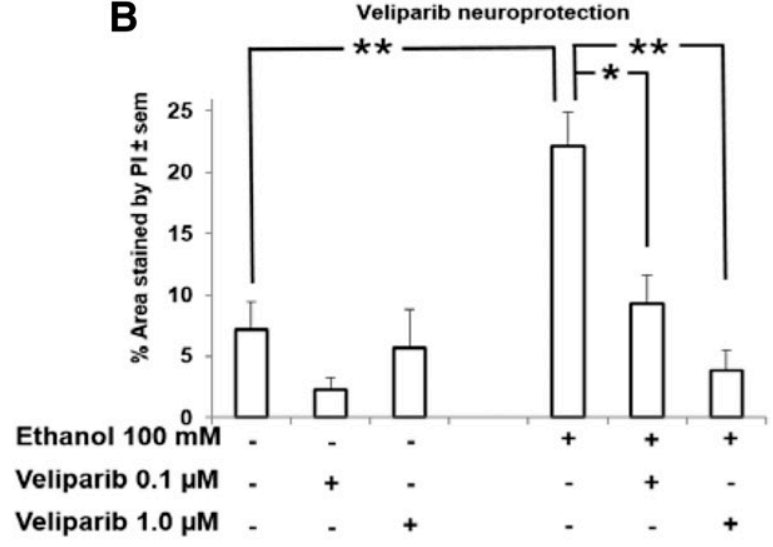

C

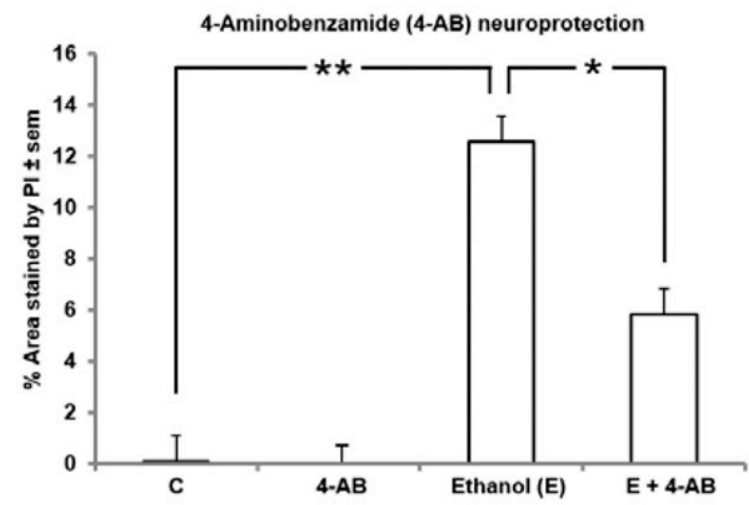

D
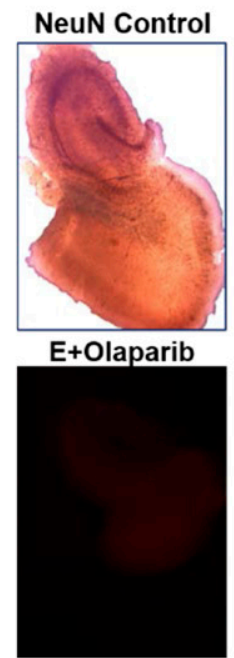

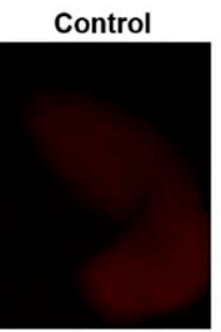

E+Veliparib

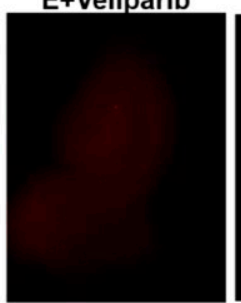

Ethanol (E)

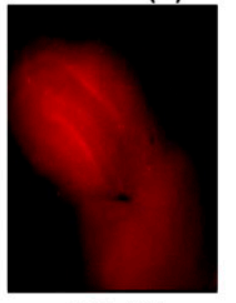

$E+4-A B$

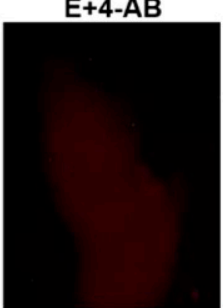

Fig. 3. Multiple PARP inhibitors prevent binge ethanol (E)-induced neurodegeneration (PI staining) in adult rat HEC slice cultures. (A) Overnight exposure of HEC slice cultures to $\mathrm{E}(100 \mathrm{mM})$ for 4 days significantly increased neurodegeneration above control PI staining, and olaparib at 0.1 or $1 \mu \mathrm{M}$ throughout prevented neurodegeneration from $\mathrm{E}$ (** $P \leq 0.01 ; n=13-20$ slices). (B) Overnight exposure of HEC slice cultures to $\mathrm{E}$ (100 mM) for 4 days significantly increased neurodegeneration above control PI staining, and veliparib at 0.1 and $1 \mu \mathrm{M}$ throughout suppressed neurodegeneration from $\mathrm{E}$ $(* P \leq 0.05 ; * * P \leq 0.01 ; n=13-25$ slices). (C) Overnight exposure of HEC slice cultures to E (100 mM) for 4 days significantly increased neurodegeneration above control PI staining, and 4-aminobenzamide (4-AB) at $500 \mu \mathrm{M}$ throughout significantly reduced neurodegeneration from $\mathrm{E}$ $(* P \leq 0.05 ; * * P \leq 0.01 ; n=10-20$ slices). (D) Representative photomicrographs of PI-stained HEC slices for control; E, $100 \mathrm{mM}$ for 4 days; E + olaparib; $\mathrm{E}+$ veliparib; and $\mathrm{E}+4$-aminobenzamide (4-AB).

(Sripathirathan et al., 2009; Tajuddin et al., 2014). As the brain's foremost water channel that is highly enriched in astroglia, AQP4 elevations have been linked to neuroinflammatory stimulation (Fukuda and Badaut, 2012). Positing that PARP activity might also promote AQP4 increases, we examined the effects of PARP inhibition on the elevated water channel levels caused by 4 days of ethanol binges. As shown in Fig. 6A, ethanol-stimulated elevations of AQP4 levels $(F(3: 21)$ $=3.62, P=0.033$ ) were not significantly affected by cotreatment with $10 \mu \mathrm{M}$ PJ34 $(P=0.82)$. Similarly, Fig. 6B shows that the significant increases in AQP4 due to ethanol $(F(5: 23)$ $=4.66, P=0.01$ ) also were not prevented by olaparib coexposure at either $0.1 \mu \mathrm{M}(P=1.0)$ or at $1 \mu \mathrm{M}(P=0.99)$.

PARP Inhibitors Prevent Ethanol-Induced Elevations of HMGB1. Since studies of ethanol's in vivo neuroinflammatory mechanisms by other laboratories have implicated HMGB1, a key brain neuroimmune agonist of TLR4 and other receptors (see Discussion), we examined whether the binge ethanol-induced significant increases of this brain alarmin in the adult HEC slice cultures shown in Fig. 1 were counteracted by PARP inhibition. Accordingly, the results shown in Fig. 7 confirm that HMGB1 levels were significantly elevated by 4 days of ethanol binges (Fig. 7A: $F(3$ : $19)=5.13, P=0.011$ and Fig. 7B: $F(3: 16)=7.57, P=0.04)$, consistent with in vivo reports by others (see Discussion). Furthermore, Fig. 7A shows that PJ34 coexposure $(10 \mu \mathrm{M})$ significantly abrogated ethanol's amplification of this brain neuroimmune proinflammatory agonist $(P=0.025)$. In addition, $1 \mu \mathrm{M}$ olaparib cotreatment completely suppressed the induction of HMGB1 by ethanol binges in the HEC slice cultures (Fig. 7B; $P=0.01$ ).

\section{Discussion}

The results indicate that PARP is an important early initiator in ethanol-binged adult brain of neuroinflammatory and neurodegenerative pathways, particularly PLA2dependent ARA cascades, but also HMGB1, which promotes 
A
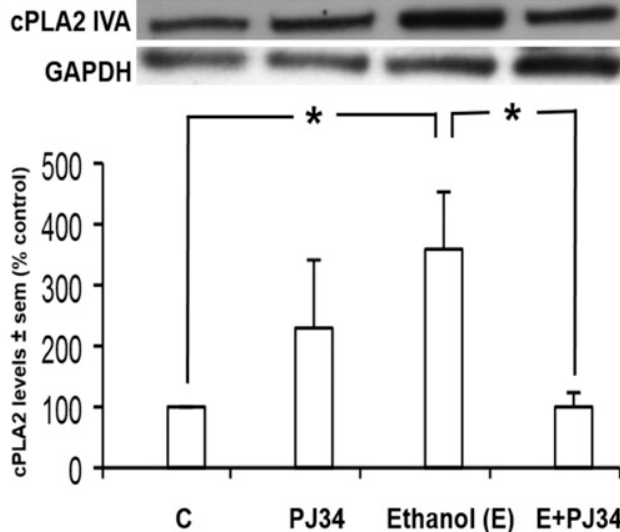

C
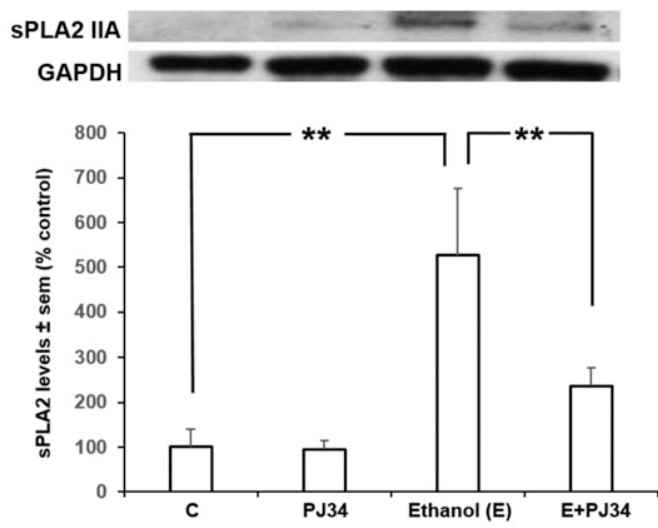

E

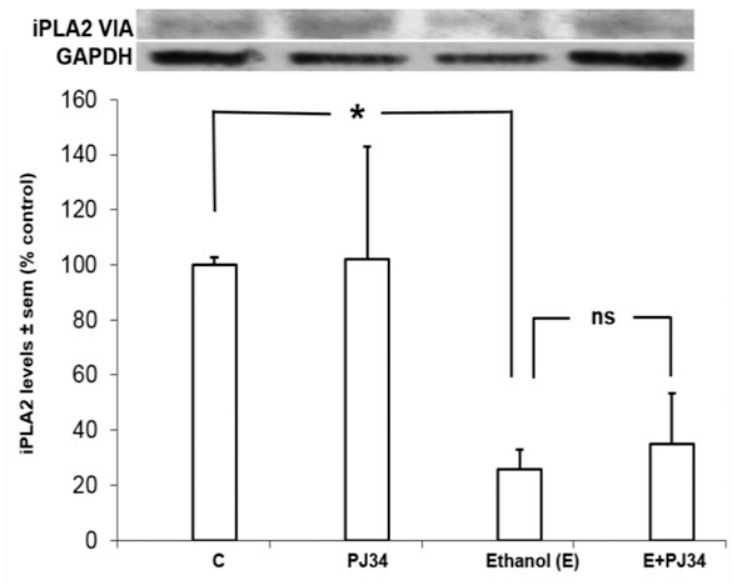

B

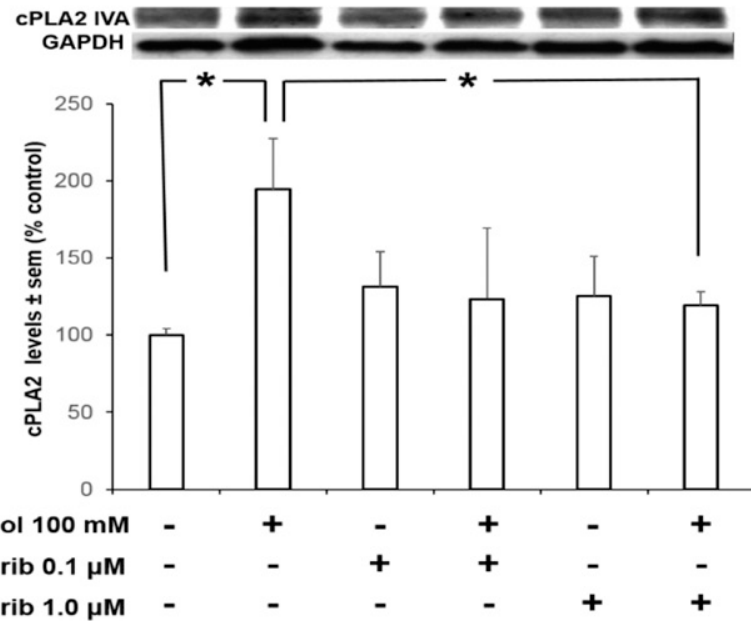

D

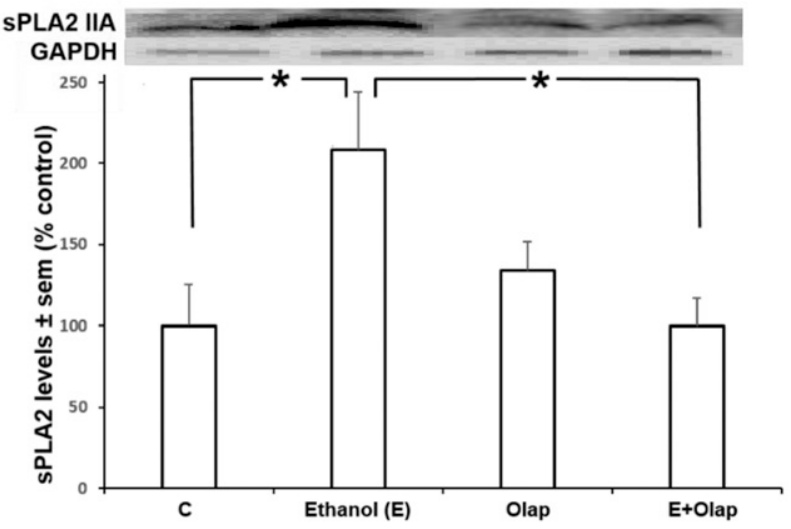

F

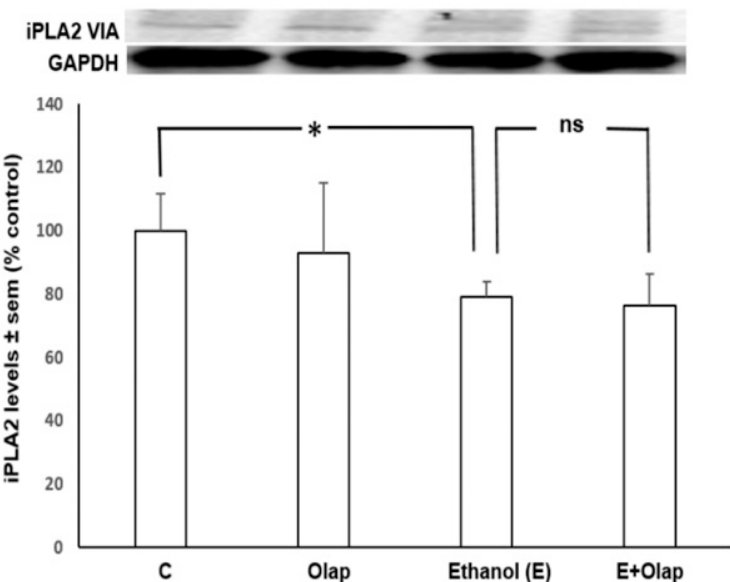

Fig. 4. PARP inhibition normalizes elevations in cPLA2 and sPLA2 levels, but not reductions of iPLA2 levels, in binge ethanol (E)-exposed adult rat HEC slice cultures. (A) Overnight exposure of HEC slice cultures to E (100 mM) for 4 days significantly elevated slice levels of cPLA2 IVA, and PJ34 $(10 \mu \mathrm{M})$ copresent throughout blocked cPLA2 increases due to $\mathrm{E}(* P \leq 0.05 ; n=5$ to 6 ). (B) Overnight exposure of HEC slice cultures to $\mathrm{E}$ (100 mM) for 4 days significantly elevated slice levels of cPLA2 IVA, and olaparib at $1 \mu \mathrm{M}$ copresent throughout blocked cPLA2 increases from $\mathrm{E}(* P \leq 0.05 ; n=3-4)$. (C) Overnight exposure of HEC slice cultures to $\mathrm{E}(100 \mathrm{mM})$ for 4 days significantly elevated slice levels of sPLA2 IIA, and PJ34 (10 $\mu \mathrm{M})$ copresent throughout prevented sPLA2 increases from $\mathrm{E}(* * P \leq 0.01 ; n=5)$. (D) Overnight exposure of HEC slice cultures to $\mathrm{E}(100 \mathrm{mM})$ for 4 days significantly augmented slice levels of sPLA2 IIA, and olaparib $(1 \mu \mathrm{M})$ copresent throughout blocked sPLA2 increases because of $\mathrm{E}(* P \leq 0.05 ; n=5)$. (E) Overnight 
A

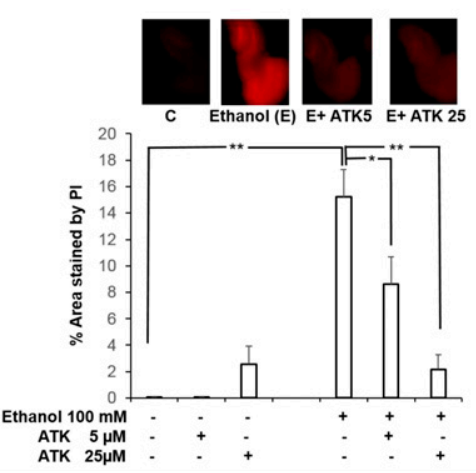

B

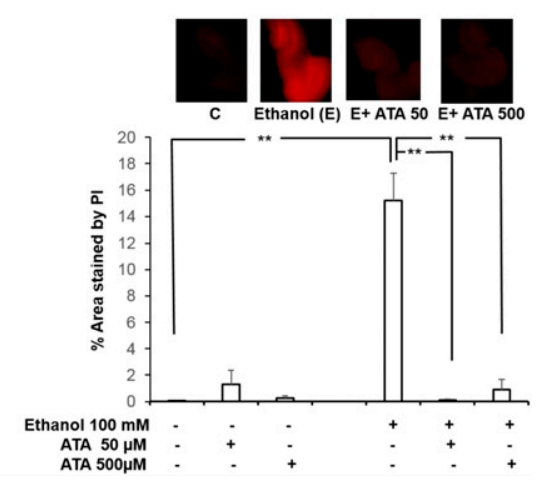

Fig. 5. Inhibition of cPLA2 with ATK or of sPLA2 with ATA prevents binge ethanol (E)-induced neurodegeneration in adult rat HEC slice cultures. (A) Overnight exposure of HEC slice cultures to E (100 mM) for 4 days significantly increased neurodegeneration above control PI staining, and ATK (5 or $25 \mu \mathrm{M}$ ) addition throughout prevented neurodegeneration resulting from $\mathrm{E}(* P \leq 0.05$ or $* * P \leq 0.01 ; n=$ 30-40 slices). (B) Overnight E treatment of HEC slice cultures for 4 days significantly increased neurodegeneration above control PI staining, and addition of ATA (50 or $500 \mu \mathrm{M})$ throughout completely protected against neurodegeneration resulting from $\mathrm{E}\left({ }^{* *} P \leq 0.01 ; n=20-30\right.$ slices). proinflammatory cytokines. Our model, long-term cultures of rat HEC slices of $\sim 60$ "brain age" days upon binge ethanol treatment, is uncommon. Brain slice cultures typically are hippocampal and early adolescent at time of treatment with ethanol or toxins (Sarnowska, 2002; Reynolds et al., 2015). Instead, we have developed slices incorporating both hippocampus and entorhinal cortex, two regions extensively neurodamaged during binge ethanol intoxication and withdrawal in adult rats (Collins et al., 1996; Obernier et al., 2002b; Kelso et al., 2011). Furthermore, although we first introduced conventional (neonatal) rat HEC slice cultures for in vitro studies of binge ethanol neurotoxicity (Collins et al., 1998), we recently perfected HEC slice cultures of young adult brain age at ethanol exposure (Collins et al., 2013) since these cultures are the more appropriate in vitro model for study of neuromechanisms in repetitively intoxicated adult rats.

In adult HEC slice cultures, immunoblots revealed binge ethanol-related PARP1 increases that are in accord with PARP1 elevations in the HEC of adult male rats subjected to binge intoxication (Tajuddin et al., 2014). PARP1, which constitutes $>90 \%$ of brain PARP, is primarily nuclear, but it is also mitochondrial (Brunyanszki et al., 2016). DNA strand breaks induce or activate the polymerase, producing PAR that recruits DNA repair molecules directly or when proteinlinked. Indeed, PARP1 "parylates" a wide array of cellular proteins, including itself (autoPARylation) (Basello and Scovassi, 2015); however, PARP hyperactivation from brain insults can precipitate regulated neuronal necrosis via PAR - that is, parthanatos (Andrabi et al., 2014; Narne et al., 2017). In experimental ischemia, brain PARP1 is elevated, and PARP inhibitors suppress necrotic neurodamage (Moroni et al., 2001; McCullough et al., 2005; Kauppinen et al., 2009). In brain trauma (TBI) models, PARP inhibition significantly attenuates neurodegeneration (Stoica et al., 2014; Tao et al., 2015). Parenthetically, binge ethanol neurodamage mechanistically mirrors TBI (Sripathirathan et al., 2009). Neuroprotection by PARP inhibitors has been reported in status epilepticus (Kim et al., 2014) and with neurotoxins-for example, soman (Meier et al., 1999), MPTP
(Yokoyama et al., 2010), and kainic acid (Mazzone and Nistri, 2011).

Several neurodamaging mechanisms may be involved in brain PARP1 hyperactivation (Conrad et al., 2016). Perhaps earliest is ATP loss and energy failure secondary to $\mathrm{NAD}^{+}$ depletion from excessive PAR formation (Ha and Snyder, 1999). Evidence also indicates that energy failure can be $\mathrm{NAD}^{+}$-independent, stemming from PAR's impairment of hexokinase, gatekeeper for glycolysis (Andrabi et al., 2014; Fouquerel et al., 2014). The mechanism for parthanatos relies on nuclear PAR translocation to mitochondria, stimulating apoptosis-initiating factor release, which promotes chromatinolytic neurodegeneration with assistance from a nuclease, macrophage-inhibiting factor (Fatokun et al., 2014; Wang et al., 2016). Cleaved PARP1 can also denote a caspasedependent apoptotic mechanism via $\sim 85-\mathrm{kD}$ fragments, but apoptosis in the Majchrowicz model is negligible (Obernier et al., 2002a).

Regardless of PARP's mechanism, our inhibitor results indicate that PARP fuels amplification of cPLA2 IVA and sPLA2 IIA, previously reported in ethanol-binged HEC slice cultures and brains of intoxicated rats (Tajuddin et al., 2014), along with consequent ARA mobilization. Published inhibitor studies of brain ischemia with aminobenzamide revealed that PARP activity also may increase inducible cyclooxygenase-2 (Koh et al., 2004), which metabolizes ARA into pro-oxidative eicosanoids. Furthermore, brains of postischemic mice lacking PAR-degrading enzyme (enhancing PAR levels above those in wild-type ischemic mice) have elevations in cyclooxygenase-2 (Cozzi et al., 2006). Our results are the first using a brain damage model showing that PARP (up)regulates the rate-controlling steps (PLA2 isoenzymes), rendering ARA available for cyclooxygenases, as well as lipoxygenases forming leukotrienes and nonenzymatic oxidations to isoprostanes, isofurans, and isoketals, concurrent with increased ROS.

Aware that chronic ethanol in vivo can increase cPLA2 activity (Hudspith et al., 1985), we were interested in cPLA2 IVA ( $~ 85 \mathrm{kDa})$ because of neuroinflammatory roles via ARA (Sun et al., 2014; Thomas et al., 2016). Furthermore, cellular

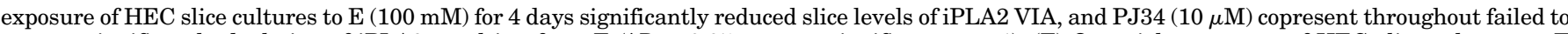

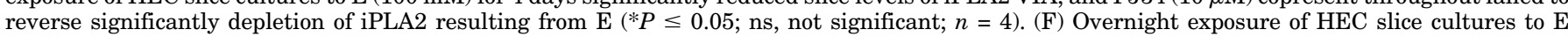

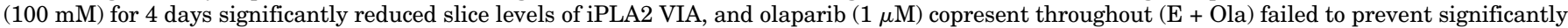
iPLA2 depletion resulting from $\mathrm{E}$ ( ${ }^{*} P \leq 0.05$; ns, not significant; $n=6$ to 7 ). 

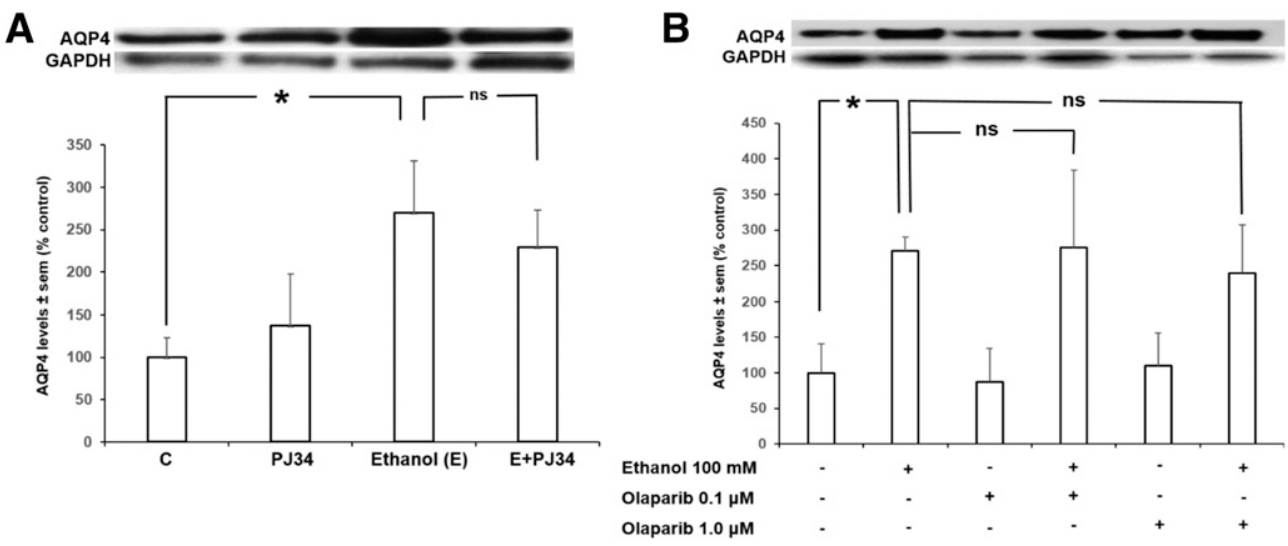

Fig. 6. PARP inhibition with PJ34 or olaparib does not block the significant augmentation of aquaporin-4 (AQP4) levels by binge ethanol (E) in adult rat HEC slice cultures. (A) Overnight exposure of HEC slice cultures to $\mathrm{E}$ $(100 \mathrm{mM})$ for 4 days increased total slice levels of AQP4, and PJ34 $(10 \mu \mathrm{M})$ copresent throughout did not significantly antagonize AQP4 elevations resulting from $\mathrm{E}(* P \leq 0.05$; ns, not significant; $n=5-7$ ). (B) Overnight exposure of HEC slice cultures to $\mathrm{E}$ $(100 \mathrm{mM})$ for 4 days amplified the total slice levels of AQP4, and olaparib (0.1 or $1 \mu \mathrm{M})$ throughout did not significantly suppress AQP4 elevations resulting from $\mathrm{E}(* P \leq 0.05 ; \mathrm{ns}$, not significant; $n=4$ ). swelling and deformation activate cPLA2 family members (Lambert et al., 2006) that respond as mechanosensitive transducers to cellular osmotic stress and membrane stretching (Lehtonen and Kinnunen, 1995; Alexander et al., 2004)and hence a relationship to binge alcohol-induced cytotoxic edema. The sPLA2 family members $(\sim 15 \mathrm{kDa}) \_$notably sPLA2 IIA - are known to be neuroinflammatory through extracellular receptor-mediated mechanisms (Moses et al., 2006), and sPLA2 inhibitors have confirmed that sPLA2 is involved in stroke and brain ischemia (Hoda et al., 2009; Chen et al., 2012). Consequently, we find that two widely used cPLA2 and sPLA2 inhibitors-ATK and ATA-neuroprotect against binge ethanol (Fig. 5), thus furnishing support for the possible mechanism of $\uparrow \mathrm{PARP} \rightarrow \uparrow \mathrm{PLA} 2 \rightarrow \uparrow \mathrm{ARA} \rightarrow \uparrow \mathrm{ROS} \rightarrow$ neurodegeneration from binge alcohol.

Whether upregulation of cPLA2 and sPLA2 by PARP is direct (e.g., PAR transcriptional role) or indirect is an unanswered issue. An indirect prospect in brain cells might be via HMGB1 since in immune cells, cPLA2 IVA activity and ARA mobilization have been connected to TLR4 activation and proinflammatory cytokines (Ruiperez et al., 2009). Also, sPLA2 IIA regulation in brain ischemia is reported to trail proinflammatory cytokine stimulation (Adibhatla and Hatcher, 2007); however, the observed time course induced by binge ethanol, with HMGB1 upregulation after cPLA2 and sPLA2 (Fig. 1), rules against the above mechanistic scenarios.
Although iPLA2 responds differently than do the $\mathrm{Ca}^{+2}$ dependent PLA2s we are studying (i.e., not significantly normalized by PARP inhibition; Fig. 4, E and F), it could nonetheless be a component in ethanol's neurodegenerative mechanism(s) owing to its depletion by ethanol (Tajuddin et al., 2014). Evidence indicates that iPLA2 activity stabilizes brain synapses and mitochondria (Allyson et al., 2012; Nordmann et al., 2014), and during neurotoxic insults that evoke elevated cPLA2, iPLA2 is decreased (Cheon et al., 2012). Also, iPLA2 regulates brain $\omega 3$ fatty acid turnoverfor example, DHA (Green et al., 2008), the loss of which is neurodeleterious, indeed, DHA is reduced by ethanol in our slices (Tajuddin et al., 2014). With respect to AQP4, the lack of the effect of PARP inhibition on ethanol's induction of the levels of this water channel is not readily explained since we previously found that pharmacologic blockade of AQP4 blockade in vivo neuroprotected against ethanol binges (Sripathirathan et al., 2009), consistent with AQP4's role in proinflammatory cytokine upregulation ( $\mathrm{Li}$ et al., 2011).

Classic cytokines have been implicated in neuroinflammatory sequelae of ethanol in animal and cell culture models (Valles et al., 2004). An ethanol-induced route stimulating proinflammatory cytokines encompasses TLR4, inflammasomes (Alfonso-Loeches et al., 2010), and endogenous HMGB1 (Crews et al., 2013; Montesinos et al., 2016). Indeed, TLR4 activation is a known neurodegenerative trigger (Lehnardt et al., 2003). A neuronal alarmin source is suggested by
A

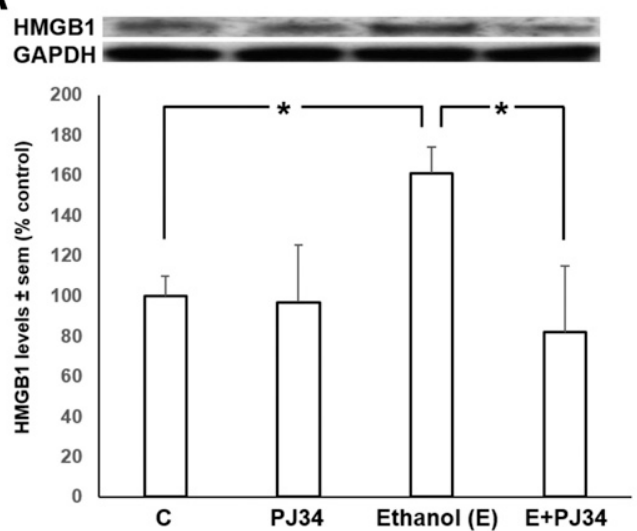

B

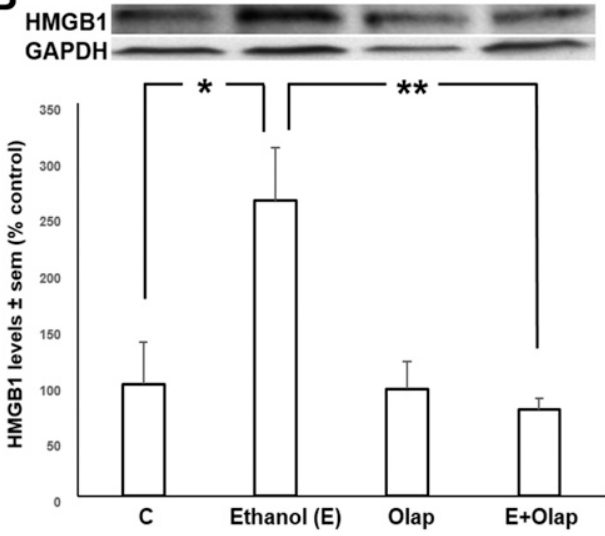

Fig. 7. PARP inhibition with PJ34 or olaparib prevents the elevations of HMGB1 levels by binge ethanol (E) in adult rat HEC slice cultures. (A) Overnight exposure of HEC slice cultures to $\mathrm{E}(100 \mathrm{mM})$ for 4 days significantly increased slice levels of total HMGB1, and cotreatment with PJ34 $(10 \mu \mathrm{M})$ throughout completely prevented HMGB1 elevation as a result of $\mathrm{E}$ (*P $\leq 0.05 ; n=6)$. (B) Overnight exposure of HEC slice cultures to E $(100 \mathrm{mM})$ for 4 days significantly increased slice levels of HMGB1, and cotreatment with olaparib $(1 \mu \mathrm{M})$ throughout significantly prevented the increase in brain slice HMGB1 resulting from $\mathrm{E}(* * P \leq 0.01$ or- $* P \leq$ $0.05 ; n=3$ or 4 ). 
evidence in neuroblastoma culture that ethanol increases or releases HMGB1 (Wang et al., 2015). Our results with two inhibitors (Fig. 7) implicate positive regulation of this neuroinflammatory alarmin by PARP, possibly driven by ethanoldependent, oxidative stress-provoked DNA strand breaks (Chiu et al., 2011). Another HMGB1 target is the receptor for advanced glycation end products (RAGE), increased in binge-intoxicated rat brains (Vetreno et al., 2013; Byun et al., 2014). This neuroinflammatory receptor is related to PARP via evidence in a stroke model that PJ34 modulates ischemiainduced rat brain RAGE (Greco et al., 2014) and that PARylation of HMGB1 in macrophages amplifies its activation of RAGE (Davis et al., 2012).

Our inhibitor results make sense if PARP activation is temporally upstream of cPLA2, sPLA2, and HMGB1, and the results shown in Fig. 1 suggest that this is so; PARP1 becomes significantly elevated after the second binge ethanol, and PAR values achieve significance by day 3 , continuing to be high on day 4. $\mathrm{Ca}^{+2}$-dependent PLA2s are elevated on day 3 and day 4, followed on day 4 by HMGB1. One speculation is that HMGB1 is dependent on a level of PARP1/PAR activity exceeding that needed to promote PLA2 isoforms, but the results also might indicate that HMGB1 requires PLA2 activity in the model.

Unlike stroke, TBI, and other acquired brain disorders, no studies have directly coupled PARP to ethanol's neurotoxicity in the adult nervous system; however, in a chronic ingestion model with adult mice (ethanol diet for $1+$ month), ethanolgenerated oxidative stress caused cerebrocortical DNA damage that was derived from one-carbon metabolism (OCM) impairment (Fowler et al., 2012). OCM is a well known metabolic process essential for DNA stability and also brain neuronal preservation. Coinciding with OCM impairment, immunocytochemical evidence was presented for increased PAR + PARylation, implicating PARP1 activity. Although differences exist between chronic liquid diet models and binge intoxication/withdrawal models that we use, it would not be remarkable if brain OCM dysfunction was involved in binge models.

As recently suggested (Berger et al., 2018), repurposing of PARP inhibitors developed for chemotherapy purposes could provide neuroprotective possibilities in acquired brain insults and chronic neurodegenerative diseases. Our in vitro results, as well as our initial in vivo results in the Majchrowicz binge model, that showed significant neuroprotection by veliparib against HEC neurodamage (Kouzoukas et al., 2017), indicate that PARP inhibition could provide brain neuroprotection in chronic alcohol abuse situations. In this context, it is noted that, although brain results were unreported, liver steatohepatitis and metabolic dysfunction were prevented by PARP inhibitors in chronic ethanol-exposed adult mice (Zhang et al., 2016; Mukhopadhyay et al., 2017).

\section{Acknowledgments}

We acknowledge the constructive criticisms and input of Dr. Dimitrios Kouzoukas and Dr. Edward J. Neafsey.

\section{Authorship Contributions}

Participated in research design: Tajuddin, Kim, Collins.

Conducted experiments: Tajuddin, Collins.

Performed data analysis: Tajuddin, Collins.

Wrote or Contributed to the writing of the manuscript: Tajuddin, Kim, Collins.

\section{References}

Adibhatla RM and Hatcher JF (2007) Secretory phospholipase A2 IIA is up-regulated by TNF-alpha and IL-1alpha/beta after transient focal cerebral ischemia in rat. Brain Res 1134:199-205.

Alexander LD, Alagarsamy S, and Douglas JG (2004) Cyclic stretch-induced cPLA2 mediates ERK 1/2 signaling in rabbit proximal tubule cells. Kidney Int 65:551-563. Alfonso-Loeches S, Pascual-Lucas M, Blanco AM, Sanchez-Vera I, and Guerri C (2010) Pivotal role of TLR4 receptors in alcohol-induced neuroinflammation and brain damage. $J$ Neurosci 30:8285-8295.

Allyson J, Bi X, Baudry M, and Massicotte G (2012) Maintenance of synaptic stability requires calcium-independent phospholipase $\mathrm{A}_{2}$ activity. Neural Plast 2012: 569149.

Andrabi SA, Umanah GK, Chang C, Stevens DA, Karuppagounder SS, Gagné JP, Poirier GG, Dawson VL, and Dawson TM (2014) Poly(ADP-ribose) polymerasedependent energy depletion occurs through inhibition of glycolysis. Proc Natl Acad Sci USA 111:10209-10214.

Basello DA and Scovassi AI (2015) Poly(ADP-ribosylation) and neurodegenerative disorders. Mitochondrion 24:56-63.

Berger NA, Besson VC, Boulares AH, Burkle A, Chiarugi A, Clark RS, Curtin NJ, Cuzzocrea S, Dawson TM, Dawson VL, et al. (2018) Opportunities for the repurposing of PARP inhibitors for the therapy of non-oncological diseases. Br J Pharmacol 175:192-222.

Brown J, III, Achille N, Neafsey EJ, and Collins MA (2009) Binge ethanol-induced neurodegeneration in rat organotypic brain slice cultures: effects of PLA2 inhibitor mepacrine and docosahexaenoic acid (DHA). Neurochem Res 34:260-267.

Brunyanszki A, Szczesny B, Virág L, and Szabo C (2016) Mitochondrial poly(ADPribose) polymerase: the Wizard of $\mathrm{Oz}$ at work. Free Radic Biol Med 100:257-270.

Byun K, Bayarsaikhan D, Bayarsaikhan E, Son M, Oh S, Lee J, Son HI, Won MH, Kim SU, Song BJ, et al. (2014) Microglial AGE-albumin is critical in promoting alcohol-induced neurodegeneration in rats and humans. PLoS One 9:e104699.

Chen S, Yao L, and Cunningham TJ (2012) Secreted phospholipase A2 involvement in neurodegeneration: differential testing of prosurvival and anti-inflammatory effects of enzyme inhibition. PLoS One 7:e39257.

Cheon Y, Kim HW, Igarashi M, Modi HR, Chang L, Ma K, Greenstein D, Wohltmann M, Turk J, Rapoport SI, et al. (2012) Disturbed brain phospholipid and docosahexaenoic acid metabolism in calcium-independent phospholipase A(2)-VIA (iPLA (2)B)-knockout mice. Biochim Biophys Acta 1821:1278-1286.

Cherian PP, Schenker S, and Henderson GI (2008) Ethanol-mediated DNA damage and PARP-1 apoptotic responses in cultured fetal cortical neurons. Alcohol Clin Exp Res 32:1884-1892.

Chiu LY, Ho FM, Shiah SG, Chang Y, and Lin WW (2011) Oxidative stress initiates DNA damager MNNG-induced poly(ADP-ribose)polymerase-1-dependent parthanatos cell death. Biochem Pharmacol 81:459-470.

Collins MA, Corso TD, and Neafsey EJ (1996) Neuronal degeneration in rat cerebrocortical and olfactory regions during subchronic "binge" intoxication with ethanol: possible explanation for olfactory deficits in alcoholics. Alcohol Clin Exp Res 20:284-292.

Collins MA, Moon KH, Tajuddin N, Neafsey EJ, and Kim HY (2013) Docosahexaenoic acid (DHA) prevents binge ethanol-dependent aquaporin-4 elevations while inhibiting neurodegeneration: experiments in rat adult-age entorhino-hippocampal slice cultures. Neurotox Res 23:105-110.

Collins MA and Neafsey EJ (2012) Neuroinflammatory pathways in binge alcoholinduced neuronal degeneration: oxidative stress cascade involving aquaporin brain edema, and phospholipase A2 activation. Neurotox Res 21:70-78.

Collins MA, Zou JY, and Neafsey EJ (1998) Brain damage due to episodic alcohol exposure in vivo and in vitro: furosemide neuroprotection implicates edema-based mechanism. FASEB J 12:221-230.

Conrad M, Angeli JP, Vandenabeele P, and Stockwell BR (2016) Regulated necrosis: disease relevance and therapeutic opportunities. Nat Rev Drug Discov 15:348-366.

Cozzi A, Cipriani G, Fossati S, Faraco G, Formentini L, Min W, Cortes U, Wang ZQ, Moroni F, and Chiarugi A (2006) Poly(ADP-ribose) accumulation and enhancement of postischemic brain damage in $110-\mathrm{kDa}$ poly(ADP-ribose) glycohydrolase null mice. J Cereb Blood Flow Metab 26:684-695.

Crews FT, Lawrimore CJ, Walter TJ, and Coleman LG, Jr (2017) The role of neuroimmune signaling in alcoholism. Neuropharmacology 122:56-73.

Crews FT, Qin L, Sheedy D, Vetreno RP, and Zou J (2013) High mobility group box $1 /$ Toll-like receptor danger signaling increases brain neuroimmune activation in alcohol dependence. Biol Psychiatry 73:602-612.

David KK, Andrabi SA, Dawson TM, and Dawson VL (2009) Parthanatos, a messenger of death. Front Biosci 14:1116-1128.

Davis K, Banerjee S, Friggeri A, Bell C, Abraham E, and Zerfaoui M (2012) Poly(ADP-ribosyl)ation of high mobility group box 1 (HMGB1) protein enhances inhibition of efferocytosis. Mol Med 18:359-369.

Ditsworth D, Zong WX, and Thompson CB (2007) Activation of poly(ADP)-ribose polymerase (PARP-1) induces release of the pro-inflammatory mediator HMGB1 from the nucleus. $J$ Biol Chem 282:17845-17854.

Fatokun AA, Dawson VL, and Dawson TM (2014) Parthanatos: mitochondrial-linked mechanisms and therapeutic opportunities. Br J Pharmacol 171:2000-2016.

Fouquerel E, Goellner EM, Yu Z, Gagné JP, Barbi de Moura M, Feinstein T, Wheeler D, Redpath P, Li J, Romero G, et al. (2014) ARTD1/PARP1 negatively regulates glycolysis by inhibiting hexokinase 1 independent of NAD + depletion. Cell Reports 8:1819-1831.

Fowler AK, Hewetson A, Agrawal RG, Dagda M, Dagda R, Moaddel R, Balbo S, Sanghvi M, Chen Y, Hogue RJ, et al. (2012) Alcohol-induced one-carbon metabolism impairment promotes dysfunction of DNA base excision repair in adult brain. $J$ Biol Chem 287:43533-43542.

Fukuda AM and Badaut J (2012) Aquaporin 4: a player in cerebral edema and neuroinflammation. J Neuroinflammation 9:279.

Gavin DP, Kusumo H, Sharma RP, and Guizzetti M (2016) Ethanol-induced changes in poly (ADP ribose) polymerase and neuronal developmental gene expression. Neuropharmacology 110 (Pt A):287-296. 
Greco R, Tassorelli C, Mangione AS, Levandis G, Certo M, Nappi G, Bagetta G, Blandini F, and Amantea D (2014) Neuroprotection by the PARP inhibitor PJ34 modulates cerebral and circulating RAGE levels in rats exposed to focal brain ischemia. Eur J Pharmacol 744:91-97.

Green JT, Orr SK, and Bazinet RP (2008) The emerging role of group VI calciumindependent phospholipase A2 in releasing docosahexaenoic acid from brain phospholipids. J Lipid Res 49:939-944.

Ha HC and Snyder SH (1999) Poly(ADP-ribose) polymerase is a mediator of necrotic cell death by ATP depletion. Proc Natl Acad Sci USA 96:13978-13982.

Hoda MN, Singh I, Singh AK, and Khan M (2009) Reduction of lipoxidative load by secretory phospholipase A2 inhibition protects against neurovascular injury following experimental stroke in rat. J Neuroinflammation 6:21.

Hudspith M, John GR, Nhamburo PT, and Littleton JM (1985) Effect of ethanol in vitro and in vivo on $\mathrm{Ca} 2+$-activated metabolism of membrane phospholipids in rat synaptosomal and brain slice preparations. Alcohol 2:133-138.

Ilomaki J, Jokanovic N, Tan EC, and Lonnroos E (2015) Alcohol consumption, dementia and cognitive decline: an overview of systematic reviews. Curr Clin Pharmacol 10:204-212

Kauppinen TM, Suh SW, Berman AE, Hamby AM, and Swanson RA (2009) Inhibition of poly(ADP-ribose) polymerase suppresses inflammation and promotes recovery after ischemic injury. J Cereb Blood Flow Metab 29:820-829.

Kelso ML, Liput DJ, Eaves DW, and Nixon K (2011) Upregulated vimentin suggests new areas of neurodegeneration in a model of an alcohol use disorder. Neuroscience 197:381-393.

Kim JE, Kim YJ, Kim JY, and Kang TC (2014) PARP1 activation/expression modulates regional-specific neuronal and glial responses to seizure in a hemodynamicindependent manner. Cell Death Dis 5:e1362.

Kinghorn KJ, Castillo-Quan JI, Bartolome F, Angelova PR, Li L, Pope S, Cochemé HM, Khan S, Asghari S, Bhatia KP, et al. (2015) Loss of PLA2G6 leads to elevated mitochondrial lipid peroxidation and mitochondrial dysfunction. Brain 138 1801-1816.

Knapp DJ and Crews FT (1999) Induction of cyclooxygenase-2 in brain during acute and chronic ethanol treatment and ethanol withdrawal. Alcohol Clin Exp Res 23 633-643.

Koh SH, Park Y, Song CW, Kim JG, Kim K, Kim J, Kim MH, Lee SR, Kim DW, Yu HJ, et al. (2004) The effect of PARP inhibitor on ischaemic cell death, its related inflammation and survival signals. Eur $J$ Neurosci 20:1461-1472.

Kouzoukas DE, Schreiber JA, Tajuddin NF, Kaja S, Kim H-Y, Neafsey EJ, and Collins MA. (2017) PARP mediates binge alcohol-induced neurodegeneration in adult rats. FASEB $J \mathbf{3 1}$ (Suppl. 1): \#661.7.

Lambert IH, Pedersen SF, and Poulsen KA (2006) Activation of PLA2 isoforms by cell swelling and ischaemia/hypoxia. Acta Physiol (Oxf) 187:75-85.

Lehnardt S, Massillon L, Follett P, Jensen FE, Ratan R, Rosenberg PA, Volpe JJ, and Vartanian T (2003) Activation of innate immunity in the CNS triggers neurodegeneration through a Toll-like receptor 4-dependent pathway. Proc Natl Acad Sci USA 100:8514-8519.

Lehtonen JY and Kinnunen PK (1995) Phospholipase A2 as a mechanosensor. Biophys $J$ 68:1888-1894.

Li L, Zhang H, Varrin-Doyer M, Zamvil SS, and Verkman AS (2011) Proinflammatory role of aquaporin-4 in autoimmune neuroinflammation. FASEB $J \mathbf{2 5}$ $1556-1566$.

Mazzone GL and Nistri A (2011) Effect of the PARP-1 inhibitor PJ 34 on excitotoxic damage evoked by kainate on rat spinal cord organotypic slices. Cell Mol Neurobiol 31:469-478.

McCullough LD, Zeng Z, Blizzard KK, Debchoudhury I, and Hurn PD (2005) Ischemic nitric oxide and poly (ADP-ribose) polymerase-1 in cerebral ischemia: male toxicity, female protection. J Cereb Blood Flow Metab 25:502-512.

Meier HL, Ballough GP, Forster JS, and Filbert MG (1999) Benzamide, a poly(ADPribose) polymerase inhibitor, is neuroprotective against soman-induced seizurerelated brain damage. Ann N Y Acad Sci 890:330-335.

Montesinos J, Alfonso-Loeches S, and Guerri C (2016) Impact of the innate immune response in the actions of ethanol on the central nervous system. Alcohol Clin Exp Res 40:2260-2270.

Moroni F, Meli E, Peruginelli F, Chiarugi A, Cozzi A, Picca R, Romagnoli P, Pellicciari R, and Pellegrini-Giampietro DE (2001) Poly(ADP-ribose) polymerase inhibitors attenuate necrotic but not apoptotic neuronal death in experimental models of cerebral ischemia. Cell Death Differ 8:921-932.

Moses GS, Jensen MD, Lue LF, Walker DG, Sun AY, Simonyi A, and Sun GY (2006) Secretory PLA2-IIA: a new inflammatory factor for Alzheimer's disease. J Neuroinflammation 3:28.

Mukhopadhyay P, Horváth B, Rajesh M, Varga ZV, Gariani K, Ryu D, Cao Z, Holovac E, Park O, Zhou Z, et al. (2017) PARP inhibition protects against alcoholic and nonalcoholic steatohepatitis. J Hepatol 66:589-600.
Narne P, Pandey V, Simhadri PK, and Phanithi PB (2017) Poly(ADP-ribose)polymerase-1 hyperactivation in neurodegenerative diseases: the death knell tolls for neurons. Semin Cell Dev Biol 63:154-166.

Nordmann C, Strokin M, Schönfeld P, and Reiser G (2014) Putative roles of $\mathrm{Ca}(2+)$ -independent phospholipase A2 in respiratory chain-associated ROS production in brain mitochondria: influence of docosahexaenoic acid and bromoenol lactone. $J$ Neurochem 131:163-176.

Obernier JA, Bouldin TW, and Crews FT (2002a) Binge ethanol exposure in adult rats causes necrotic cell death. Alcohol Clin Exp Res 26:547-557.

Obernier JA, White AM, Swartzwelder HS, and Crews FT (2002b) Cognitive deficits and CNS damage after a 4-day binge ethanol exposure in rats. Pharmacol Biochem Behav 72:521-532.

Reynolds AR, Berry JN, Sharrett-Field L, and Prendergast MA (2015) Ethanol withdrawal is required to produce persisting N-methyl-D-aspartate receptordependent hippocampal cytotoxicity during chronic intermittent ethanol exposure. Alcohol 49:219-227.

Ruipérez V, Astudillo AM, Balboa MA, and Balsinde J (2009) Coordinate regulation of TLR-mediated arachidonic acid mobilization in macrophages by group IVA and group V phospholipase A2s. J Immunol 182:3877-3883.

Sachdeva A, Chandra M, Choudhary M, Dayal P, and Anand KS (2016) Alcoholrelated dementia and neurocognitive impairment: a review study. Int $J$ High Risk Behav Addict 5:e27976.

Sarnowska A (2002) Application of organotypic hippocampal culture for study of selective neuronal death. Folia Neuropathol 40:101-106.

Sripathirathan K, Brown J, III, Neafsey EJ, and Collins MA (2009) Linking binge alcoholinduced neurodamage to brain edema and potential aquaporin-4 upregulation: evidence in rat organotypic brain slice cultures and in vivo. $J$ Neurotrauma 26:261-273.

Stoica BA, Loane DJ, Zhao Z, Kabadi SV, Hanscom M, Byrnes KR, and Faden AI (2014) PARP-1 inhibition attenuates neuronal loss, microglia activation and neurological deficits after traumatic brain injury. J Neurotrauma 31:758-772.

Sun GY, Chuang DY, Zong Y, Jiang J, Lee JC, Gu Z, and Simonyi A (2014) Role of cytosolic phospholipase A2 in oxidative and inflammatory signaling pathways in different cell types in the central nervous system. Mol Neurobiol 50:6-14.

Tajuddin N, Moon KH, Marshall SA, Nixon K, Neafsey E.J, Kim HY, and Collins MA (2014) Neuroinflammation and neurodegeneration in adult rat brain from binge ethanol exposure: abrogation by docosahexaenoic acid. PLoS One 9:e101223.

Tajuddin NF, Przybycien-Szymanska MM, Pak TR, Neafsey EJ, and Collins MA (2013) Effect of repetitive daily ethanol intoxication on adult rat brain: significant changes in phospholipase A2 enzyme levels in association with increased PARP-1 indicate neuroinflammatory pathway activation. Alcohol 47:39-45.

Tao X, Chen X, Hao S, Hou Z, Lu T, Sun M, and Liu B (2015) Protective actions of PJ34, a poly(ADP-ribose)polymerase inhibitor, on the blood-brain barrier after traumatic brain injury in mice. Neuroscience 291:26-36.

Thomas MH, Pelleieux S, Vitale N, and Olivier JL (2016) Dietary arachidonic acid as a risk factor for age-associated neurodegenerative diseases: potential mechanisms. Biochimie 130:168-177.

Vallés SL, Blanco AM, Pascual M, and Guerri C (2004) Chronic ethanol treatment enhances inflammatory mediators and cell death in the brain and in astrocytes. Brain Pathol 14:365-371.

Vetreno RP, Qin L, and Crews FT (2013) Increased receptor for advanced glycation end product expression in the human alcoholic prefrontal cortex is linked to adolescent drinking. Neurobiol Dis 59:52-62.

Wang Y, An R, Umanah GK, Park H, Nambiar K, Eacker SM, Kim B, Bao L, Harraz MM, Chang C, et al. (2016) A nuclease that mediates cell death induced by DNA damage and poly(ADP-ribose) polymerase-1. Science 354:aad6872.

Wang X, Chu G, Yang Z, Sun Y, Zhou H, Li M, Shi J, Tian B, Zhang C, and Meng X (2015) Ethanol directly induced HMGB1 release through NOX2/NLRP1 inflammasome in neuronal cells. Toxicology 334:104-110.

Yang Z, Li L, Chen L, Yuan W, Dong L, Zhang Y, Wu H, and Wang C (2014) PARP-1 mediates LPS-induced HMGB1 release by macrophages through regulation of HMGB1 acetylation. J Immunol 193:6114-6123.

Yokoyama H, Kuroiwa H, Tsukada T, Uchida H, Kato H, and Araki T (2010) Poly(ADP-ribose)polymerase inhibitor can attenuate the neuronal death after 1-methyl4-phenyl-1,2,3,6-tetrahydropyridine-induced neurotoxicity in mice. $J$ Neurosci Res 88:1522-1536.

Zhang Y, Wang C, Tian Y, Zhang F, Xu W, Li X, Shu Z, Wang Y, Huang K, and Huang $\mathrm{D}$ (2016) Inhibition of poly(ADP-ribose) polymerase-1 protects chronic alcoholic liver injury. Am J Pathol 186:3117-3130.

Address correspondence to: Michael A. Collins, Department of Molecular Pharmacology and Therapeutics, Loyola University Chicago, Stritch School of Medicine, Maywood, IL 60153. E-mail: mcollin@luc.edu 